\title{
A Study on Structural Design and Analysis of Blade and Tower for Small Wind Turbine System
}

\author{
Hyunbum Park ${ }^{1, a}$ \\ ${ }^{1}$ Department of Defense Science \&Technology - Aeronautics, Howon University, 54058, 64 Howondae 3gil, Impi, Gunsan, Rep. of Korea
}

\begin{abstract}
This paper performed aerodynamic, structural design and analysis of the horizontal axis blade of low noise $1 \mathrm{~kW}$ class small wind turbine system that can be operated at homes and designed to display excellent performances in low wind velocity regions such as Korea. In terms of the material applied, glass/epoxy that is both economical and excellent in its performance was applied. Structural test was conducted to verify whether the actual movement of the blade designed is consistent with the theoretical analysis result. In addition, structural safety review was performed through the structural analysis of a tower for installing the wind turbine system developed.
\end{abstract}

\section{Introduction}

Although wind power energy has been continuously used since ancient times, wind turbine system is being researched and developed in various ways recently in the midst of a current situation where studies are being actively conducted on alternative energy development resulting from the depletion of fossil fuel. Currently, a significant amount of power supply is being substituted through wind power generation centering on Europe and it is said that over $10 \%$ is being covered in regions such as Denmark and Northern Germany [1]. In terms of the research trend of wind power generation, wind turbine systems are being developed in various scales from a small system to a large power generation system. Since a small wind turbine system can be operated independently in a small scale, related studies are being actively conducted in recent [2]. In addition, the use of composite materials has led to the result of a significant improvement of structural strength and fatigue life. However, majority of the blade of small wind turbine system has been developed in advanced countries and the rated wind speed of imported blades is not suitable for the domestic climate condition. Accordingly, there is a need to develop wind turbine system suitable for the domestic climate condition.

This paper performed aerodynamic, structural design and analysis of the horizontal axis blade of low noise $1 \mathrm{~kW}$ class small wind turbine system that can be operated at homes and designed to display excellent performances in low wind velocity regions such as Korea. In terms of the material applied, glass/epoxy that is both economical and excellent in its performance was applied. Structural test was conducted to verify whether the actual movement of the blade designed is consistent with the theoretical analysis result. In addition, structural safety review was performed through the structural analysis of a tower for installing the wind turbine system developed.

\section{Blade Design}

For the purpose of designing wind turbine system, it is necessary to first specify the system specification. As for the specification of the wind turbine system in this paper, it is horizontal axis wind turbine system with rate output of $1 \mathrm{~kW}$, rated wind velocity of $12.8 \mathrm{~m} / \mathrm{s}$ and applied airfoil NACA $632-615$. The blade diameter is $2.54 \mathrm{~m}$ and angle of torsion is $22.6^{\circ}$. The turbine system to be applied is a direct-driven AFPM (axial flux permanent magnet) turbine system that can be applied as a highefficiency energy conversion device even at low wind velocity.

Structural design was performed after blade aerodynamic design to evaluate the structural safety of blade through structural analysis and fatigue life analysis. Structural load for structural design includes the aerodynamic load applied to blade and centrifugal load as main load.

Based on the structural design load, skin-spar-foam sandwich structure was adopted as the structural form of blade for the design. In the blade structural design form, skin and spar are stacked in the blade longitudinal with foam applied inside the blade, and it was designed to reduce the number of layers through longitudinal from the root part of blade for weight reduction by calculating section-specific load.

For the structural analysis conducted to evaluate the structural safety of structural design result, finite element commercial code was used, and Tsai-Wu failure criterion [3] was applied as the failure criterion for the safety review. The result of linear static analysis for each load

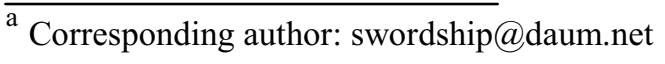


condition confirmed that it has been designed with a safe structure ensuring sufficient safety factor. Natural frequency was analyzed to examine any resonance of blade and the result showed that resonance did not occur around operational number of rotations. The result of buckling stability evaluation also confirmed that it is a structure ensuring a sufficient level stability during operation with over 4 times the load. The maximum fatigue load calculated based on Spera's empirical formula for fatigue life analysis was $576.6 \mathrm{Nm}$ and the analysis result of stress on fatigue load showed maximum compressive stress of $35.4 \mathrm{MPa}$ and maximum tensile stress of $28.0 \mathrm{MPa}$, thereby confirming that a sufficient level of safety rate has been ensured regarding allowable fatigue strength (144.7MPa). Accordingly, the blade design result confirmed that it is a structure that ensured required fatigue life of 20 years.

The structural analysis result was compared with the final structural test result for verification and they were consistent. The linear static analysis result and fatigue life analysis result were specified respectively in Fig. 1.

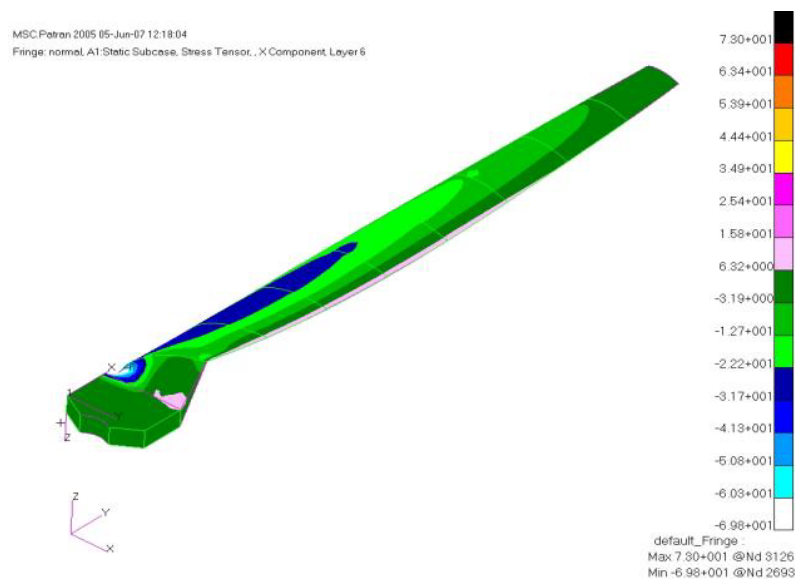

Figure 1. Stress analysis result of designed blade.

\section{Tower Design}

Structural analysis was performed for the tower that supports the blade system of this study to evaluate its structural safety [4]. The structural analysis of tower was performed by first determining load by considering each load condition and analyzing stress and displacement analysis after verifying the validity of load. As for load condition according to each situation, load of tower can be considered according to wind velocity changes. In this paper, two types of cases were considered with the load placed on tower during normal operation (load case 1) and the load placed on tower when it stopped during a storm (load case 2). Specific loads that need to be considered during tower structural analysis are load from thrust generated as blade rotates, and distributed load placed on tower from wind and load placed from nacelle $\&$ blade and tower weight they can be considered as non uniform load with concentrated load placed in the upper part of tower and distributed load placed on the entire tower. Fig. 2 shows the entire shape of tower. The structural analysis results showed that stress placed on tower during normal operation is $23.4 \mathrm{MPa}$ and the stress on tower when it stopped during a storm is $154 \mathrm{MPa}$, thereby confirming that it is a structure that ensured a sufficient level of operational safety. Fig. 3 and 4 shows the results of structural analysis for each load condition and Fig. 6 is a view of blade and wind turbine system manufactured in this paper installed in a tower.

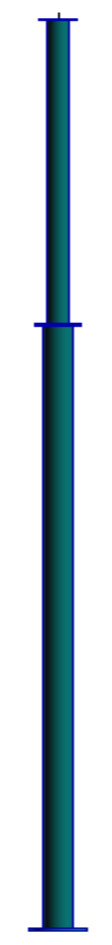

Figure 2. Structural configuration of automobile bonnet panel.

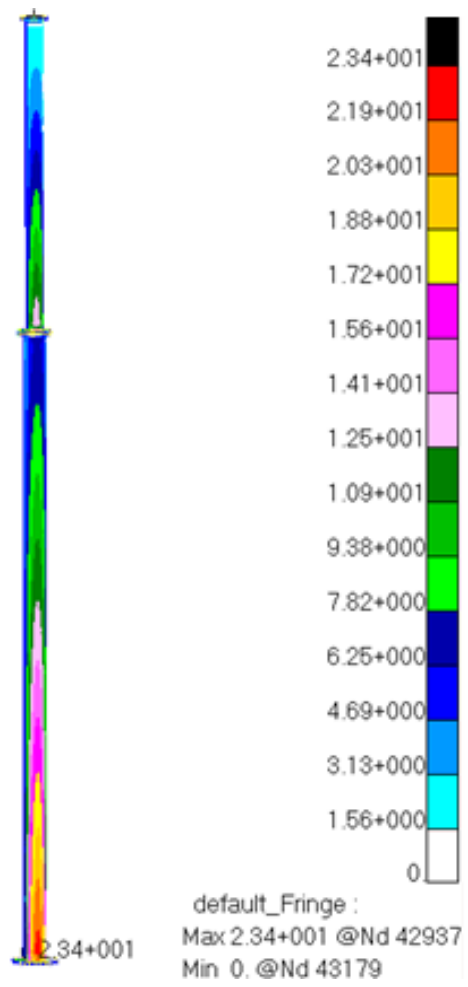

Figure 3. Stress contour of load case 1. 

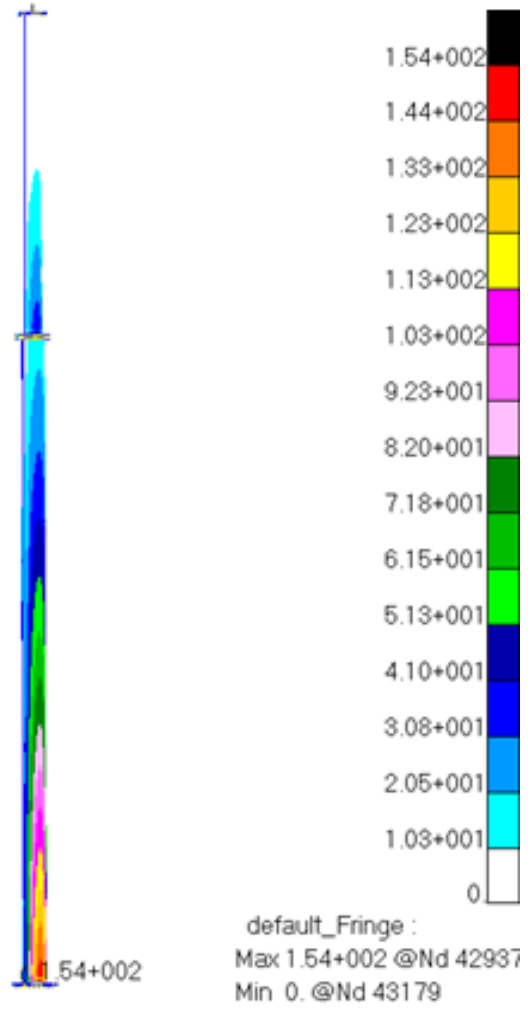

Figure 4. Stress contour of load case 2.

\section{Conclusions}

This paper performed aerodynamic, structural design and analysis of the horizontal axis blade of low noise $1 \mathrm{~kW}$ class small wind turbine system that can be operated at homes and suitable for domestic regions. The result of reviewing its structural safety based on structural analysis showed that it is a structure that ensured a sufficient level of safety. In addition, the result of fatigue life analysis showed that it sufficiently satisfied the required life of 20 years. Based on the structural analysis of a tower supporting the blade system, it was confirmed that the tower structural design is a structure that ensured a sufficient level of safety.

\section{Acknowledgments}

This research was supported by Basic Science Research Program through the National Research Foundation of Korea (NRF) funded by the Ministry of Education (No. 2014R1A1A2054842). This study was supported by Howon University research funds.

\section{References}

1. D. LE Gourieres, Wind power plants, (Pergamon Press, 1982)

2. Changduk Kong, Johyuk Bang, Kyungwon Oh, Structural Design of $1 \mathrm{~kW}$ class small wind turbine blade, Proceeding of Fall Conference on The Korean Society of Aeronautical \& Space Science, (2003)
3. Fu-Kuo Chang, Richard A. Scott, George S. Springer, Strength of Mechanically Fastened Composite Joints, Journal of Composite Materials, 16 (1982)

4. Changduk Kong, Structural Design of Medium Scale Composite Wind Turbine Blade, Proceeding of $13^{\text {th }}$ International Conference on Composite Materials, (2001). 